\title{
Interference Assisted Laser Induced Forward Transfer for Structured Patterning
}

\author{
Ankur Shah, Avinash Parashar, Jasjit Singh Mann and Narayanswamy Sivakumar \\ Department of Mechanical and Industrial Engineering, EV 13-310, Concordia University, 1515 St. Catherine West, \\ Montreal, Quebec, H3G 2W1, Canada
}

\begin{abstract}
Structured line patterns find a great deal of application in the field of microelectronics and optoelectronic devices. In this communication, a new method for the deposition of the periodic structured patterns with nanosecond pulsed laser interference together with Laser Induced Forward Transfer (LIFT) has been presented. Laser Induced Forward Transfer is a simple direct write technique to micro pattern a surface by localized deposition of material. Patterns transferred were evaluated with the help of optical microscopy and atomic force microscopy images. The main advantage of this method is that we can modify the pattern dimensions by simple changes in the optical setup.
\end{abstract}

\section{INTRODUCTION}

In the past few years, research on the fabrication of periodic line patterns has found a growing interest. Such structured patterns find applications in the field of BioMEMS [1], contact mask making [2] electronic devices and integrated optics [3]. Direct writing technologies have emerged as an important tool, in which structures are built directly without the use of masks which also allow rapid prototyping of devices. Laser Induced Forward Transfer (LIFT) is one such direct write technique where the laser radiation passes through an optically transparent donor substrate and is focused at the interface of donor substrate and thin absorbing film. The film ablated from the donor will get deposited on an acceptor substrate kept in close proximity to donor $[4,5]$.

Periodic patterns with LIFT can also be created by using principle of interference [2]. One such attempt has been made by Quan-Zhong Zhao et al. in their research paper [6], in which multiple laser beam were interfered for creating regular dot patterns on glass substrate. So far no attempt has been made for creating periodic line patterns with LIFT. To address these issues work has been done with interference based LIFT and the results are summarized in this communication.

\section{EXPERIMENTAL SETUP}

The optical setup shown in Fig. (1) has been modified from work described in [7]. In the modified setup beam spacing between parallel beams can be easily managed with different set of diverging lenses, whereas previously it was done with a different alignment of Wollaston's which is tedious. Nd: YVO4 laser (1064nm) with pulse width of $14 \mathrm{~ns}$, and tunable repetition rate was used in all the experiments. Gold film of $50 \mathrm{~nm}$ thickness was ablated from glass donor and deposited on acceptor glass substrate. All the

*Address correspondence to this author at the Department of Mechanical and Industrial Engineering, EV 13-310, Concordia University, 1515 St. Catherine West, Montreal, Quebec, H3G 2W1, Canada;

E-mail: ankur_832001@yahoo.com experiments were done in ambient conditions with donor film and acceptor substrate kept in contact mode.

\section{EXPERIMENTS AND DISCUSSION}

Experiments were done with different pulse energies ranging from $30 \mu \mathrm{J}$ to $280 \mu \mathrm{J}$ and at repetition rate of 20 $\mathrm{kHz}$. The ablated and deposited gold patterns are shown in Figs. $(2,3)$ respectively. It can be observed from Fig. (3) that with increase in pulse energy deposition also increases. The quality of the deposited gold fringe patterns increases with increase in the pulse energy which is clearly evident from deposition done above $44 \mu \mathrm{J}$.

A study was done in order to evaluate percentage deposition of patterns with respect to laser energy to optimize the quality of deposition. Percentage deposition is the ratio of deposited lines to that of the ablated lines. Fig. (4) shows the variation of deposition of the fringe patterns with energy.

It can be seen from the graph that number of deposited line patterns increases with increase in energy. In nanosecond laser, film removal is explosive in nature shown in Fig. (5) where the laser energy is used to shear the film from the donor substrate and deposit on to the acceptor [8]. Considering the Gaussian beam distribution, at higher energies, the corners of the beam has the energy to deposit the line patterns thereby increasing the efficiency. Good quality of deposited pattern is seen in energies ranging from $30 \mu \mathrm{J}$ to $170 \mu \mathrm{J}$. Though greater deposition efficiency can be achieved at energies above $170 \mu \mathrm{J}$; the quality starts diminishing and the deposited gold film spreads outside of the focused spot area, possibly because of transition to boiling phase [9] as shown in Fig. (6).

\section{QUALITY OF DEPOSITION: AFM ANALYSIS}

Pulse energy has significant effect on the quality of the fringe pattern deposition. The quality of the patterns deposited has been discussed with the help of Atomic Force Microscopy results as shown in Fig. (7). Patterns deposited were homogenous and uniform for energies in the range of 


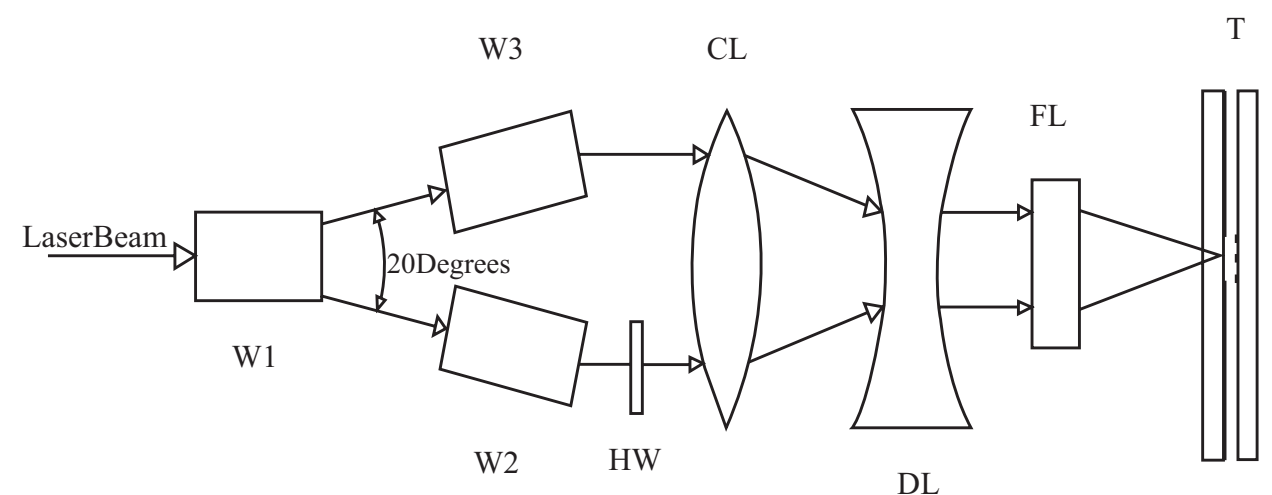

Fig. (1). W1 - Wollaston Prism 1; W2 - Wollaston Prism 2; W3 - Wollaston Prism 3 ; HW - Half Wave Plate; FL - Focusing Lens; CL Converging Lens; DL - Diverging Lens; T - Target (Donor substrate, Acceptor substrate and thin film).

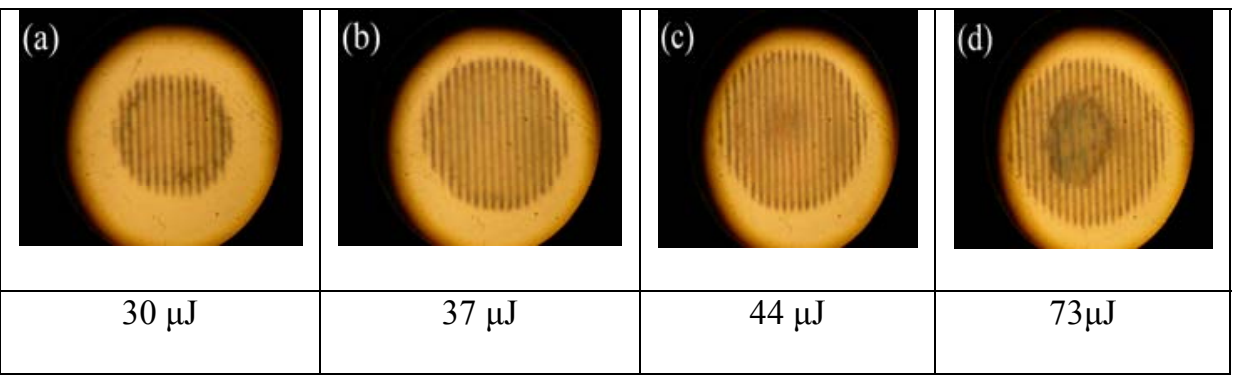

Fig. (2). Gold fringe patterns ablated from donor glass between $(30 \mu \mathrm{J}-80 \mu \mathrm{J})$, f.o.v $100 \mu \mathrm{m}$.

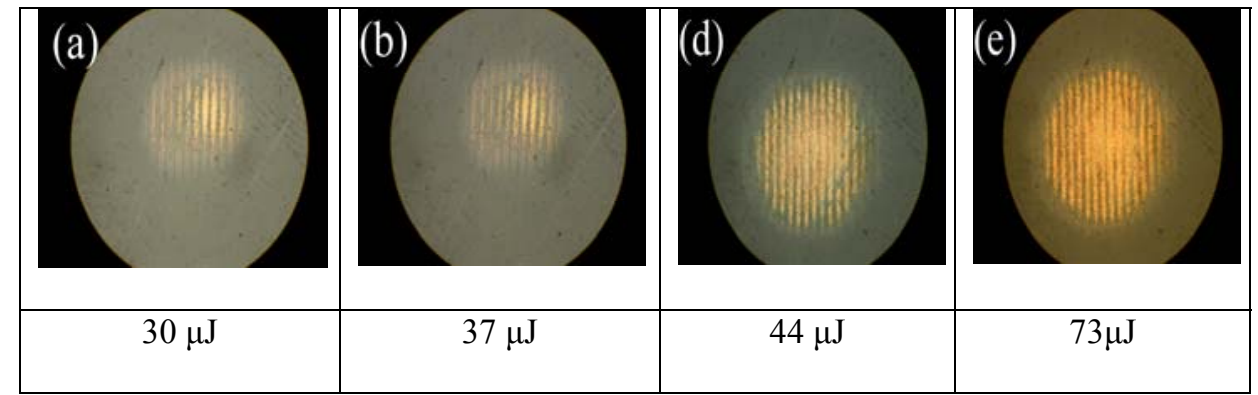

Fig. (3). Gold fringe patterns deposited on acceptor substrate between $(30 \mu \mathrm{J}-80 \mu \mathrm{J})$, f.o.v $100 \mu \mathrm{m}$.

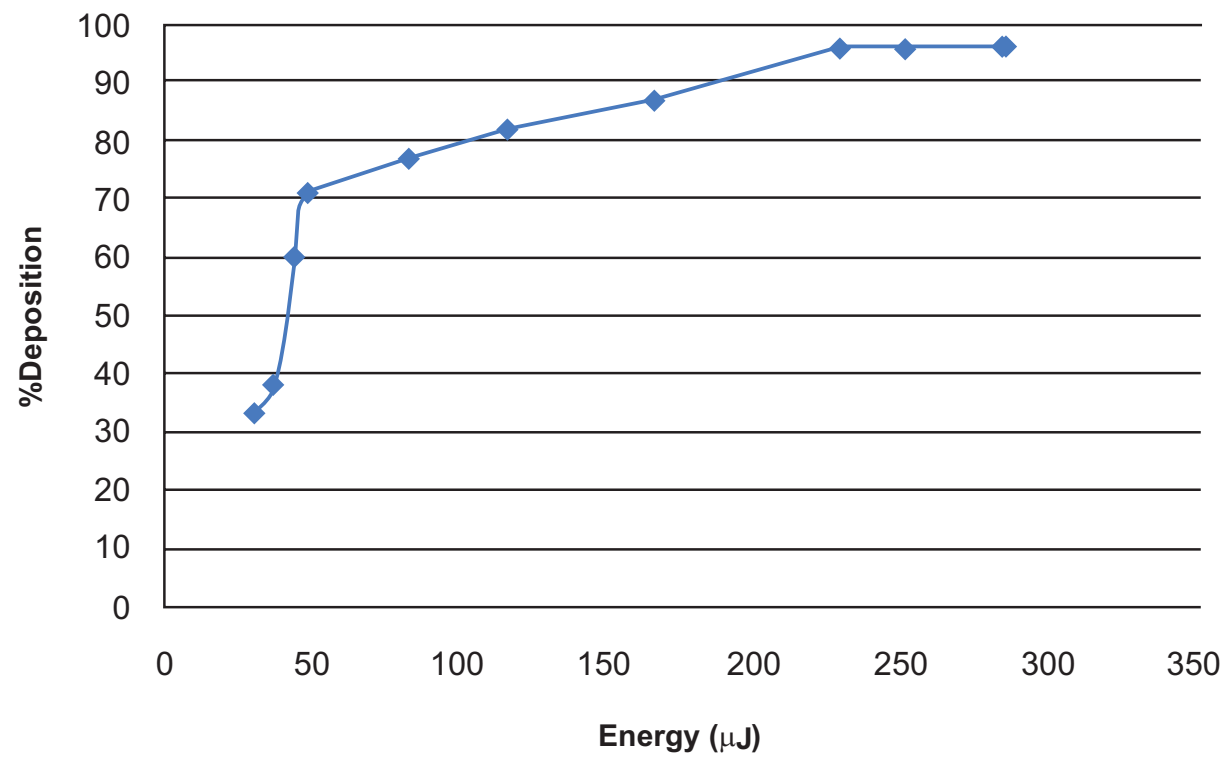

Fig. (4). Variation in deposition of gold line patterns with energy. 


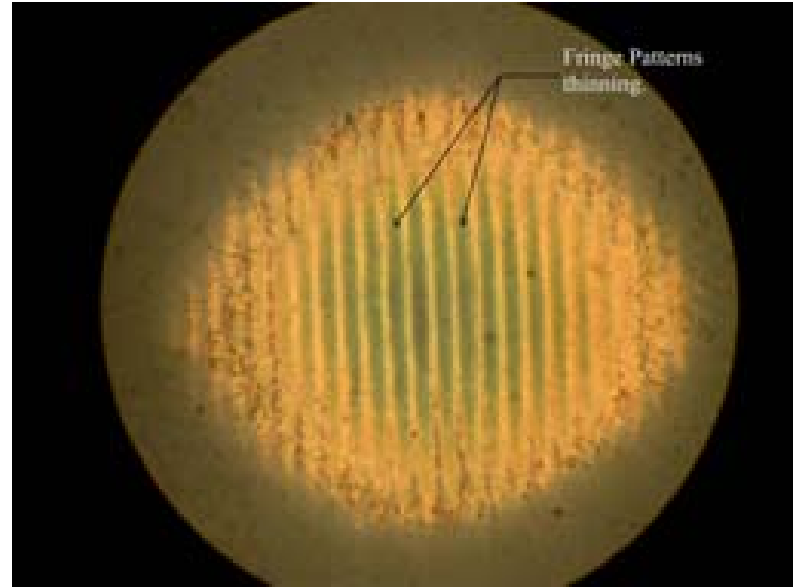

Fig. (5). Deposited gold fringe pattern thinning at $225 \mu \mathrm{J}$, f.o.v $100 \mu \mathrm{m}$.

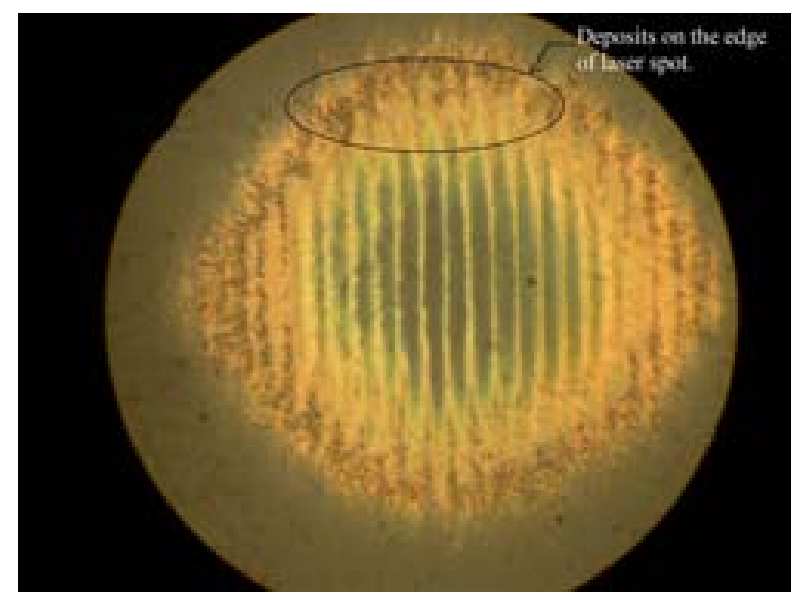

Fig. (6). Gold material deposition on the periphery of the deposited pattern at $250 \mu \mathrm{J}$.
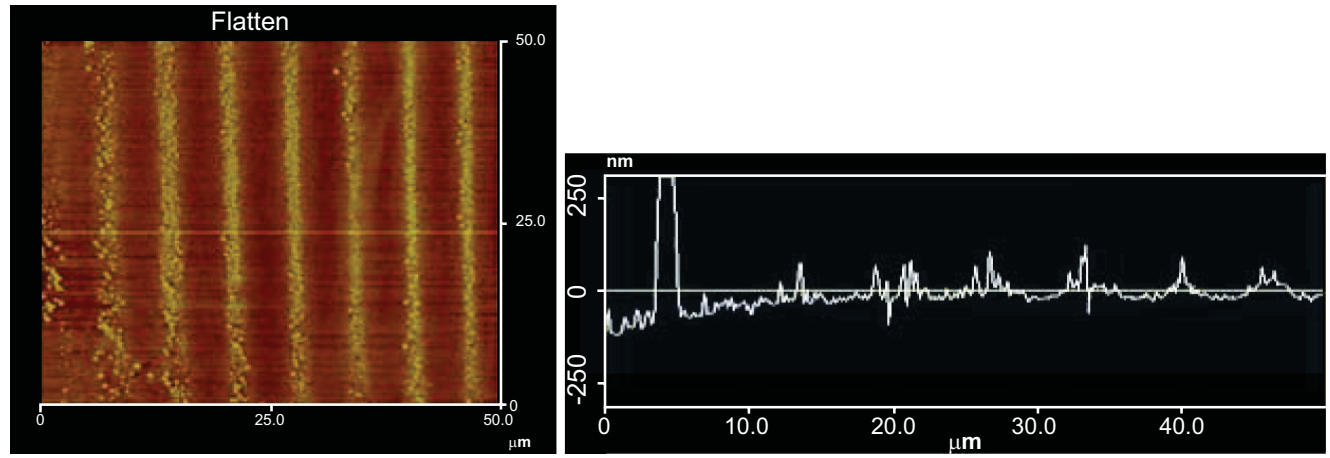

Fig. (7). AFM image showing fringe pattern deposition at $73 \mu \mathrm{J}$.

$30 \mu \mathrm{J}-170 \mu \mathrm{J}$. For higher pulse energies the gold spattered and the imprints become nonuniform. The effect of Gaussian beam intensity on the deposited pattern can be clearly seen from the Fig. (7). On the edges of the spot the gold has just spattered around while in the central region uniform deposition is observed.

Study has been done with interference based LIFT for structured line pattern deposition of Gold that has various applications. Experiments were done with different pulse energies to identify the optimum laser parameter for quantitative and qualitative deposition of gold fringe patterns.

\section{ACKNOWLEDGEMENTS}

The authors would like to acknowledge NSERC (Natural Sciences and Engineering Research Council) of Canada and the CFI (Canada Foundation for Innovation) for the funding support provided.

\section{REFERENCES}

[1] Wang W, Soper SA, Eds. Bio-MEMS: Technologies and Applications. USA: CRC Press 2006.

[2] Landström L, Klimstein J, Schrems G, Piglmayer K, Bäuerle D. Single-step patterning and the fabrication of contact masks by laser-induced forward transfer. J Appl Phys A 2004; 78(4): 537.

[3] Hussell CP, Ramaswamy RV. High-index overlay for high reflectance DBR gratings in $\mathrm{LiNbO} 3$ channel waveguides. IEEE Photonic Technol Lett 1997; 9(5): 636.

[4] Bohandy J, Kim BF, Adria FJ. Metal deposition from a supported metal film using an excimer laser. J Appl Phys1986; 60:1538-9.

[5] Toth Z, Szorenyi T, Toth AL. Ar+ laser-induced forward transfer (LIFT): a novel method for micrometer-size surface patterning. Appl Surf Sci 1993; 69: 317-20.

[6] Zhao Q-Z, Qiu J-R, Zhao C-J, Jiang X-W, Zhu C-S. Optical transfer of periodic microstructures by interfering femtosecond laser beams. Optics Express 2005; 13: 1304-3109.

[7] Venkatakrishnan K, Sivakumar NR, Tan B. Direct grating writing using femtosecond laser interference fringes formed at the focal point. J Opt A: Pure Appl Opt 2005; 7: 169-74.

[8] Yamada H, Sano T, Nakayama T, Miyamoto I. Optimization of laser-induced forward transfer process of metal thin films. Appl Surf Sci 2002; 197: 411-15.

[9] Miotello A, Kelly R. Critical assessment of thermal models for laser sputtering at high fluences. Appl Phys Lett 1995; 67(24): 3535. 